\title{
NOTE ON DEHN FILLINGS CREATING MÖBIUS BANDS AND KLEIN BOTTLES
}

\author{
SEungsang OH
}

\begin{abstract}
In this paper we investigate the distances between Dehn fillings on a hyperbolic 3-manifold that yield 3-manifolds containing a Möbius band and a Klein bottle.
\end{abstract}

\section{Introduction}

Let $M$ be a compact, connected, orientable 3-manifold with a torus boundary $T$. A slope on $T$ is the isotopy class of an essential simple loop. If $\gamma$ is a slope on $T$, then as usual we denote by $M(\gamma)$ the 3-manifold obtained by $\gamma$-Dehn filling on $M$, that is, attaching a solid torus $V_{\gamma}$ to $M$ along $T$ in such a way that $\gamma$ bounds a disk in the torus.

In the present paper we assume that $M\left(\gamma_{1}\right)$ contains a Möbius band and $M\left(\gamma_{2}\right)$ contains a Klein bottle. Suppose that $M\left(\gamma_{i}\right)$ contains such a surface $\widehat{F}_{i}$ for $i=1,2$. Then we may assume that $\widehat{F}_{i}$ meets the attached solid torus $V_{\gamma_{i}}$ in a finite collection of meridian disks, and is chosen so that the number of disks $n_{i}$ is minimal among all such surfaces in $M\left(\gamma_{i}\right) . \Delta\left(\gamma_{1}, \gamma_{2}\right)$ denotes their minimal geometric intersection number. This paper gives the proof of the following theorem mostly based on the results of Lee, Oh and Teragaito [2].

Theorem 1. Suppose that $M$ is hyperbolic. If $M\left(\gamma_{1}\right)$ contains a Möbius band and $M\left(\gamma_{2}\right)$ contains a Klein bottle, then $\Delta\left(\gamma_{1}, \gamma_{2}\right) \leq 3$ unless $n_{1}=1$ or $n_{2}=1$.

Let $F_{i}=\widehat{F}_{i} \cap M$. By an isotopy we may assume that $F_{1} \cap F_{2}$ has the minimal number of components and they intersect transversely. Then we obtain graphs $G_{i}$ in $\widehat{F}_{i}$ as usual. We use the definitions and terminology of [2]. An $x$-edge in $G_{i}$ is an edge with label $x$ at one endpoint, and level $x$-edge is a positive edge with label $x$ at both endpoints. A vertex $x$ is

Received February 2, 2010. Accepted March 10, 2010.

Key words and phrases: Dehn filling, essential annulus, Klein bottle.

This work was supported by the National Research Foundation of Korea(NRF) grant funded by the Korea government (MEST) (No. 2009-0074101). 
called a level vertex if $x$ is the label of a level edge on the other graph. The following lemmas are Lemmas 2.4., 2.5. and 2.6. in that paper.

Lemma 2. $G_{1}$ has at most one level vertices and $G_{2}$ has at most two level vertices. Furthermore both $G_{i}$ cannot have a generalized $S$-cycle.

Lemma 3. $G_{1}$ has at most $\frac{n_{2}}{2}+1$ mutually parallel positive edges and $n_{2}$ mutually parallel negative edges. Similarly $G_{2}$ has at most $\frac{n_{1}+1}{2}$ mutually parallel positive edges and $n_{1}$ mutually parallel negative edges.

\section{2. $x$-face and extremal block}

A disk face of the subgraph of $G_{i}$ consisting of all the vertices and positive $x$-edges is called an $x$-face. The following theorem is Theorem 3.4. in [2].

Theorem 4. $G_{2}$ cannot contain an $x$-face where $x$ is not the level vertex of $G_{1}$.

The reduced graph $\bar{G}_{1}$ of $G_{1}$ is defined to be the graph obtained from $G_{1}$ by amalgamating each family of parallel edges into a single edge. Let $G_{1}^{+}$denote the subgraph of $G_{1}$ consisting of all vertices and positive edges of $G_{1}$. For convenience, cap off the boundary of $\widehat{F}_{1}$ by a disk, which is called a fake disk. Then we think of $G_{1}$ as a graph in the projective plane. Then each component of $G_{1}^{+}$has a disk support because any orientation-preserving loop in a projective plane is contractible. A disk support which possibly contains the fake disk is called a fake disk support

Lemma 5. Each non-level vertex of $G_{1}$ has at least $\left(\Delta\left(\gamma_{1}, \gamma_{2}\right)-1\right) n_{2}$ positive edge endpoints.

Proof. Assume that there is a non-level vertex $x$ of $G_{1}$ which has more than $n_{2}$ negative edges. Then $G_{2}$ contains more than $n_{2}$ positive $x$-edges by the parity rule. Thus the subgraph $\Gamma_{x}$ of $G_{2}$ consisting of all vertices and positive $x$-edges has more edges than vertices. Then an Euler characteristic calculation shows that $\Gamma_{x}$ contains a disk face, which is an $x$-face. This contradicts Theorem 4 .

From now on, we assume that $\Delta \geq 4$ and $n_{1} \geq 2$. Thus $G_{1}$ has non-level vertices. Note that each of these vertices has therefore valency at least two in $\bar{G}_{1}$ by Lemmas 3 . Now take an innermost component $\Lambda_{0}$ of $G_{1}^{+}$with a fake disk support $D_{0}$, which means that $D_{0} \cap G_{1}^{+}=\Lambda_{0}$.

Suppose that $\Lambda_{0}$ is a single vertex. Since all positive edges are mutually parallel loops, this vertex has at most $n_{2}+2$ positive edge endpoints 
by Lemma 3. By Lemma 5, it must be a level vertex. So a negative loop is incident there. Thus we can also choose another innermost component of $G_{1}^{+}$which contains only non-level vertices.

We may therefore assume that $\Lambda_{0}$ has more than one vertex. Then $\Lambda_{0}$ has either no cut vertex or at least two blocks with at most one cut vertex. Thus we can choose an innermost component $\Lambda$ with a fake disk support $D$ after splitting $\Lambda_{0}$ at all cut vertices, such that $\Lambda$ has more than one vertex with at most one cut vertex. If it is the case that $\Lambda$ contains a cut vertex and a distinct level vertex, we can choose another innermost one containing no level vertex.

Such a subgraph $\Lambda$ of $G_{1}^{+}$is called an extremal block with a fake disk support $D$. A vertex of $\Lambda$ is called a ghost vertex if it is either a cut vertex or a level vertex. We emphasize that $\Lambda$ has more than one vertex and at most one ghost vertex $y_{0}$. A vertex of $\Lambda$ is called a boundary vertex if there is an arc connecting it to $\partial D$ whose interior is disjoint from $\Lambda$, and an interior vertex otherwise. Then the preceding argument together with Lemma 5 proves the following theorem, which plays a key role in this paper:

Lemma 6. Suppose that $\Delta \geq 4, n_{1} \geq 2$ and $n_{2} \geq 2$. $G_{1}$ contains an extremal block $\Lambda$ with a fake disk support $D$ so that each boundary vertex excluding $y_{0}$ has at least $3 n_{2}$ consecutive edge endpoints of $\Lambda$.

\section{Proof of Theorem 1}

Assume that $\Delta\left(\gamma_{1}, \gamma_{2}\right) \geq 4, n_{1} \geq 2$ and $n_{2} \geq 2$. As mention in [2], $M\left(\gamma_{2}\right)$ is irreducible and boundary irreducible.

Lemma 6 says that $G_{1}^{+}$contains an extremal block $\Lambda$ with a fake disk support $D$ so that each boundary vertex, except $y_{0}$, has at least $3 n_{2}$ consecutive edge endpoints of $\Lambda$. First, assume that $n_{2}=2$. This case is done by the similar argument of $[1$, Section 6$]$.

Assume that $n_{2} \geq 3$. Choose a non-level label $x$ of $G_{1}$ by Lemma 2 . Let $\Lambda^{x}$ be the subgraph of $\Lambda$ consisting of all vertices and $x$-edges. Then each boundary vertex of $\Lambda^{x}$, except $y_{0}$, has at least three edges attached with label $x$, and any two among them cannot be parallel by Lemma 3 . Note that $\Lambda^{x}$ may not be connected. Then, apply the argument in Section 2 to the present situation; choose an extremal block $\Lambda^{\prime}$ of $\Lambda^{x}$ with a fake disk support $D^{\prime}$ in $D$, which we can define in a similar way.

Let $v, e$ and $f$ be the numbers of vertices, edges, and disk faces of $\Lambda^{\prime}$, respectively. Also let $v_{i}, v_{\partial}$ and $v_{g}$ be the numbers of interior vertices, 
boundary vertices and ghost vertices. Hence $v=v_{i}+v_{\partial}$ and $v_{g}=0$ or 1.

Suppose that $\Lambda^{\prime}$ has a bigon which does not contain the fake disk. By Lemma 3, it contains a generalized $S$-cycle. But this is impossible by Lemma 2. Thus, except at most one, each face of $\Lambda^{\prime}$ is a disk with at least 3 sides. Hence we have $3 f+v_{\partial}-2 \leq 2 e$. Since $\Lambda^{\prime}$ has only disk faces, combined with $v-e+f=\chi\left(D^{\prime}\right)=1$, we get $e \leq 3 v_{i}+2 v_{\partial}-1$. On the other hand, we have $3\left(v_{\partial}-v_{g}\right)+4 v_{i} \leq e$, because each boundary vertex of $\Lambda^{\prime}$, except $y_{0}$, has at least three edges attached with label $x$, and $x$ is not a label of level edges. These two inequalities give us that $v_{i}+v_{\partial}+1 \leq 3 v_{g}$, so $v_{g}=1$ and $v_{i}+v_{\partial} \leq 2 . \Lambda^{\prime}$ may have one interior vertex and one boundary vertex, which is a ghost vertex. Now the boundary vertex which is not the ghost vertex has at least three edges attached with label $x$ connecting both vertices. Even if one face contains the fake disk, some two of these $x$-edges are parallel through another face, a contradiction.

This completes the theorem.

\section{References}

1. G. T. Jin, S. Lee, S. Oh and M. Teragaito, $P^{2}$-reducing and toroidal Dehn fillings, Math. Proc. Camb. Philos. Soc. 134 (2003), 271-288.

2. S. Lee, S. Oh and M. Teragaito, Reducing Dehn fillings and small surfaces, Proc. London Math. Soc. (3) 92 (2006), 203-223.

\section{Seungsang Oh}

Department of Mathematics, Korea University, Anam-dong, Sungbuk-ku, Seoul 136-701, Korea

E-mail: seungsang@korea.ac.kr 